ИЗВЕСТИЯ АКАДЕМИИ НАУК ЭСТОНСКОИ ССР. ТОМ 28 ФИЗИКА * МАТЕМАТИКА, 1979, № 2

\title{
ГРУППОВОЙ ПОДХОД В ДИНАМИКЕ МНОГОСПИНОВЫХ СИСТЕМ. VI
}

(Представлена Э. Липпмаа)

Тема данной статьи - динамика абстрактной трехуровневой системы не исчерпывается изложенным. Здесь рассматривается широкий класс пропагаторов, построенных в виде произведения трех экспонент, и дается перечень более простых поддинамик, описываемых пропагаторами представлений группы $S U(2)$ в группе $S U(3)$.

\section{0. Динамика трехуровневой системы}

10.1. Супероператорное представление группы $\mathbf{u}_{3}$. Выберем совокупность собственных векторов $a_{m}(m=1,2,3)$ статического гамильтониана $H_{0}$ изучаемой системы в качестве исходного базиса 3-мерного пространства C чистых квантовых состояний. Ей соответствует эрмитовый $A$-базис (базисные операторы $A_{m m}, X_{m n}, Y_{m n}$ ) 9-мерного пространства $\mathbf{H}$ всех эрмитовых операторов $\left[{ }^{1}\right]$. На $A_{m m}$ натянуто 3-мерное коммутативное пространство $\mathbf{H}_{A} \subset \mathbf{H}$, содержащее $H_{0}$. Так как $\operatorname{tr} H_{0}=0$, то $H_{0}$ локализован в 2-мерном подпространстве $\mathbf{H}_{A}{ }^{0} \subset \mathbf{H}_{A}$. Ортогональным базисом для $\mathbf{H}_{A}{ }^{0}$ могут служить операторы

$$
\begin{aligned}
& Z_{m n}=(1 / 2)\left(A_{m m}-A_{n n}\right), \\
& Q_{m n}=(2 / 3)\left(Z_{m k}-Z_{k n}\right) .
\end{aligned}
$$

Отметим, что скалярные произведения

$$
\begin{gathered}
\left(Z_{m n}, Z_{m n}\right)=\left(X_{m n}, X_{m n}\right)=\left(Y_{m n}, Y_{m n}\right)=1 / 2, \\
\left(Q_{m n}, Q_{m n}\right)=2 / 3 .
\end{gathered}
$$

Собственным векторам $a_{m}, a_{k}, a_{n}$ соответствуют собственные значения оператора $Z_{m n}(+1 / 2,0,-1 / 2)$ и собственные значения оператора $Q_{m n}(+1 / 3,-2 / 3$, $+1 / 3)$.

Поскольку имеется три перехода $m \rightarrow n$, следовательно, существует и три типа базисов (10.1) и (10.2). Ниже будет показано в качестве типичного примера разложение $H_{0} \in \mathbf{H}_{A}{ }^{0}$ по базису перехода $2 \rightarrow 3$

$$
H_{0}=\omega_{L}^{0} Z_{23}+\omega_{S}^{0} Q_{23} \text {, }
$$

где

$$
\omega_{L}^{0}=\omega_{23}^{0}=\omega_{2}^{0}-\omega_{3}^{0} \text {, }
$$




$$
\omega_{s}^{0}=(1 / 2)\left(\omega_{21}^{0}-\omega_{13}^{0}\right),
$$

а $\omega_{m}^{0}$ - собственное значение оператора $H_{0}$.

Согласно Ф. Д. Мойнихену $\left[{ }^{2}\right]$, любой $U \in \mathbf{u}_{3}$ может быть представлен в виде произведения

$$
U=U_{A} T \text {, }
$$

где

$$
\begin{gathered}
U_{A}=\exp \left[-i \sum_{m} \alpha_{m} A_{m m}\right], \\
T=T_{23}\left(\varphi_{23}, \sigma_{23}\right) T_{12}\left(\varphi_{12}, \sigma_{12}\right) T_{13}\left(\varphi_{13}, \sigma_{13}\right), \\
T_{m n}\left(\varphi_{m n}, \sigma_{m n}\right)=\exp \left[-i 2 \varphi_{m n} Y_{m n}\left(\sigma_{m n}\right)\right] .
\end{gathered}
$$

Входящие в (10.10) угловые параметры $\varphi_{m n}, \sigma_{m n}$ группы $\mathbf{u}_{3}$ могут изменяться в пределах

$$
\begin{gathered}
-\pi \leqslant \varphi_{13}, \varphi_{23} \leqslant \pi, \\
-\pi / 2 \leqslant \varphi_{12}, \sigma_{m n} \leqslant \pi / 2 .
\end{gathered}
$$

Между угловыми параметрами $\alpha_{m}$ существует зависимость

$$
\sum_{m} \alpha_{m}=0,
$$

обеспечивающая унимодулярность оператора $U_{A}$.

Пара операторов $X_{m n}\left(\sigma_{m n}\right), Y_{m n}\left(\sigma_{m n}\right)$ получается путем «правого поворота» $X_{m n} \rightarrow Y_{m n}$ пары $X_{m n}, Y_{m n}$ на угол $\sigma_{m n}$. Сокращенно:

$$
X_{m n} \stackrel{\sigma_{m n}}{\longrightarrow} Y_{m n}
$$

С помощью той же сокращенной символики действие супероператора $\mathfrak{2}_{m n}\left(\varphi_{m n}, \sigma_{m n}\right)$ оператора $(10.11)$ на элементы $A$-базиса записывается так:

$$
\begin{aligned}
& Z_{m n} \stackrel{2 \Phi_{m n}}{\longrightarrow} X_{m n}\left(\sigma_{m n}\right), \\
& X_{m k}(\sigma) \stackrel{\Phi_{m n}}{\longrightarrow} X_{k n}(\sigma), \\
& Y_{m k}(\sigma) \stackrel{\Phi_{m n}}{\longleftarrow} Y_{k n}(\sigma) .
\end{aligned}
$$

Действие $\widetilde{I}_{m n}\left(\varphi_{m n}, \sigma_{m n}\right)$ в натянутом на $X_{m k}, Y_{m k}, X_{k n}, Y_{k n}$ подпространстве $\mathbf{H}(m k, k n)$ не зависит от угла $\sigma_{m n}$. Поэтому не только $X_{m k}, X_{k n}$ и $Y_{m k}, Y_{k n}$, но и любые $X_{m k}(\sigma), X_{k n}(\sigma)$ и $Y_{m k}(\sigma), Y_{k n}(\sigma)$ являются плоскостями вращения.

Несколько слов о порядке индексов $m, n$ операторов $X_{m n}, Y_{m n}$. В конкретных случаях придерживаемся порядка $m<n$. При переходе от общих формул к конкретным следует учитывать, что

$$
X_{n m}=X_{m n}, \quad Y_{n m}=-Y_{m n} .
$$

Унитарное преобразование

$$
e_{m}^{(3)}=T a_{m}
$$

переводит $a_{m}$ в новый базис $e_{m}^{(3)} \in \mathbf{C}$, имеющий некоторую «унитарную ориентацию» относительно «системы отсчета» $a_{m}$. Унитарная ориентация определяется шестью параметрами $\varphi_{m n}, \sigma_{m n}$ оператора $(10.10)$. Соответствующий $(10.10)$ супероператор $\mathfrak{2}$ переводит $A$-базис в новый 
$E^{(3)}$-базис пространства $\mathbf{H}$ (базисные операторы: $\left.E_{m m}^{(3)}, X_{m n}^{(3)}, Y_{m n}^{(3)}\right)$. В частности, подпространство $\mathbf{H}_{A}^{0}$ переводится в подпространство $\mathbf{H}_{F 3}^{0}$, его базис типа $(10.1),(10.2)$ обозначаем как $Z_{m n}^{(3)}, Q_{m n}^{(3)}$.

Ниже будут применены три базиса $e_{m}^{(j)}(j=1,2,3)$ вместе с соответствующими $E^{(j)}$-базисами и подпространствами $\mathbf{H}_{F j}^{0}$ : Мы их определяем согласно формулам

где

$$
\begin{aligned}
& e_{m}^{(1)}=T_{23} a_{m}, \\
& e_{m}^{(2)}=T_{12}^{\prime} e_{m}^{(1)}, \\
& e_{m}^{(3)}=T_{13}^{\prime \prime} e_{m}^{(2)},
\end{aligned}
$$

$$
\begin{gathered}
T_{12}^{\prime}=T_{23} T_{12} T_{23}^{-1}, \\
T_{13}^{\prime \prime}=\left(T_{23} T_{12}\right) T_{13}\left(T_{23} T_{12}\right)^{-1} .
\end{gathered}
$$

Так как

$$
T=T_{13}^{\prime \prime} T_{12}^{\prime} T_{23},
$$

то $(10.20)$ согласуется с $(10.23)$.

Итак, $\widetilde{T}_{12}^{\prime}$ совершает преобразование типа $(10.16)-(10.18)$ над $E^{(1)}$-базисом. При этом $m n=12$ (см. табл. 1). Аналогично, $\mathfrak{I}_{13}{ }^{\prime \prime}$ преобразует $E^{(2)}$-базис в $E^{(3)}$-базис (см. табл. 2 ).

Таблица 1

\begin{tabular}{c|c|c}
\hline Операторы $E^{(1)}$-базиса & Операторы $A$-базиса & $\begin{array}{c}\text { Угол } \\
\text { поворота }\end{array}$ \\
\hline$Z_{23}^{(1)}, X_{23}^{(1)}\left(\sigma_{23}\right)$ & $Z_{23}, X_{23}\left(\sigma_{23}\right)$ & $2 \varphi_{23}$ \\
$X_{12}^{(1)}, X_{13}^{(1)}$ & $X_{12}, X_{13}$ & $\varphi_{23}$ \\
$Y_{12}^{(1)}, Y_{13}^{(1)}$ & $Y_{12}, Y_{13}$ & $\varphi_{23}$ \\
$Q_{23}^{(1)}=Q_{23}$ & & $Y_{23}^{(1)}\left(\sigma_{23}\right)=Y_{23}\left(\sigma_{23}\right)$
\end{tabular}

Таблица 2

$E^{(2)}$-базис пространства $\mathbf{H}$ на языке $E^{(1)-б а з и с а ~(п р е о б р а з о в а н и е ~} \mathfrak{Z}_{12}^{\prime}$ )

\begin{tabular}{c|c|c}
\hline Операторы $E^{(2)}$-базиса & Операторы $E^{(1)}$-базиса & $\begin{array}{c}\text { Угол } \\
\text { поворота }\end{array}$ \\
\hline$Z_{12}^{(2)}, X_{12}^{(2)}\left(\sigma_{12}\right)$ & $Z_{12}^{(1)}, X_{12}^{(1)}\left(\sigma_{12}\right)$ & $2 \varphi_{12}$ \\
$X_{13}^{(2)}, X_{23}^{(2)}$ & $X_{13}^{(1)}, X_{23}^{(1)}$ & $\varphi_{12}$ \\
$Y_{13}^{(2)}, Y_{23}^{(2)}$ & $Y_{13}^{(1)}, Y_{23}^{(1)}$ & $\varphi_{12}$ \\
& & $Y_{12}^{(2)}\left(\sigma_{12}\right)=Y_{12}^{(1)}\left(\sigma_{12}\right)$
\end{tabular}


Разъясним смысл сокращенной записи, например, в табл. 1. По данным второго ряда имеем

$$
\begin{aligned}
& X_{12}^{(1)}=\cos \varphi_{23} X_{12}+\sin \varphi_{23} X_{13}, \\
& X_{13}^{(1)}=-\sin \varphi_{23} X_{12}+\cos \varphi_{23} X_{13},
\end{aligned}
$$

а последний ряд показывает, что 2-мерное подпространство над $Q_{23}, Y_{23}\left(\sigma_{23}\right)$ не изменяется под влиянием $\mathfrak{I}_{23}$.

Преобразование $\widetilde{I}_{23}$ базиса $Q_{23}, Z_{23}$ подпространства $\mathbf{H}_{A}{ }^{0}$ приводит к базису $Q_{23}, Z_{23}{ }^{(1)}$ подпространства $\mathbf{H}_{F 1}{ }^{0}$. Совершая с помощью формул

$$
\begin{aligned}
& Q_{23}^{(1)}=-(1 / 2) Q_{12}^{(1)}-Z_{12}^{(1)}, \\
& Z_{23}^{(1)}=(3 / 4) Q_{12}^{(1)}-(1 / 2) Z_{12}^{(1)}
\end{aligned}
$$

переход к базису $Q_{12}^{(1)}, Z_{12}^{(1)}$ того же подпространства, сможем удобным образом применять преобразование $\widetilde{I}_{12}^{\prime}$ K $E^{(1)}$-базису. Аналогично, преобразование базисов подпространства $\mathbf{H}_{F 2}^{0}$

$$
\begin{aligned}
& Q_{13}^{(2)}=-(1 / 2) Q_{12}^{(1)}+Z_{12}^{(2)}, \\
& Z_{13}^{(2)}=(\overline{3} / 4) Q_{12}^{(1)}+(1 / 2) Z_{12}^{(2)}
\end{aligned}
$$

позволяет прилагать $\mathfrak{T}_{13}^{\prime \prime}$ к $E^{(2)}$-базису, чтобы получить $E^{(3)}$-базис. К этому базису относятся, в частности, операторы

$$
\begin{aligned}
& Q_{13}^{(3)}=Q_{13}^{(2)}, \\
& Z_{13}^{(3)}=\cos 2 \varphi_{13} Z_{13}^{(2)}+\sin 2 \varphi_{13} X_{13}^{(2)}\left(\sigma_{13}\right),
\end{aligned}
$$

составляющие базис подпространства $\mathbf{H}_{F 3}^{0}$.

В подпространстве $\mathbf{H}_{F 3}^{0}$ локализованы все эрмитовые операторы, унитарная ориентация которых задана шестью параметрами $\varphi_{m n}, \sigma_{m n}$. В частности, эти параметры и «естественные популяции» $\pi_{m}$, определяемые по

$$
\varrho(0) e_{m}^{(3)}=\pi_{m} e_{m}^{(3)},
$$

задают любое начальное состояние $\varrho(0)$ оператора плотности $\varrho(t)$ изучаемой системы. Стало быть,

$$
\varrho(0)=(1 / 3) E+\varrho_{S}(0) Q_{13}^{(2)}+\varrho_{L}(0) Z_{13}^{(2)} \in \mathbf{H}_{F 3}^{0},
$$

где

$$
\begin{aligned}
& \varrho_{L}(0)=\pi_{13}=\pi_{1}-\pi_{3}, \\
& \varrho_{S}(0)=(1 / 2)\left(\pi_{12}-\pi_{23}\right) .
\end{aligned}
$$

Относительно $E^{(2)-б а з и с а ~ и м е е м ~}$

$$
\begin{aligned}
\varrho(0) & =(1 / 3) E+\left[(3 / 4) \varrho_{L}(0) \cos 2 \varphi_{13}-(1 / 2) \varrho_{S}(0)\right] Q_{12}^{(1)}+ \\
& +\left[(1 / 2) \varrho_{L}(0) \cos 2 \varphi_{13}+\varrho_{S}(0)\right] Z_{12}^{(2)}+ \\
& +\varrho_{L}(0) \sin 2 \varphi_{13} X_{13}^{(2)}\left(\sigma_{13}\right) .
\end{aligned}
$$

1,0.2. Групповой подход. Перечислим методические принципы 
группового подхода [1 $\left.{ }^{1}\right]$ применительно к динамике трехуровневой системы (см. также $\left.\left[{ }^{3}\right]\right)$.

1. Компактное описание движения в пространстве $\mathbf{C}$ достигается применением пропагаторов $D(t, 0) \in \mathbf{u}_{3}$. Их супероператоры $\Re(t, 0)$ определяют целые семейства траекторий:

$$
\varrho(t)=\Re(t, 0) \varrho(0) .
$$

Каждый $\Re(t, 0)$ соответствует некоторому гамильтониану $H(t) \in \mathbf{u}_{3}{ }^{0}$. 2. Основная задача динамики сводится тем самым к установлению соответствий

$$
H(t)=H_{0}+H_{E}(t) \leftrightarrow D(t, 0) \rightarrow \Re(t, 0) .
$$

3. Если входящие в (10.36) величины выражены на языке $A$-базиса, то преобразования типа (10.35) описывают динамику абстрактной трехуровневой системы. Получается зависимость $\Re(t, 0)$ от возбуждения $H_{E}(t)$, заданного относительно $H_{0}$ как характеристики системы.

4. Если рассматриваемая трехуровневая система является ядерным спином 1 , то установление соответствия между $A$-базисом и составленным из спиновых тензоров $I$-базисом $\left[{ }^{1}\right]$ позволяет дать физическую интерпретацию выводам абстрактной динамики.

5. Представления динамической группы $\mathbf{u}_{2}$ (двухуровневой системы) над группой $\mathbf{u}_{3}$ описывают более простые поддинамики трехуровневой системы. Перечень этих поддинамик дан в п. 10.3 .

6. Пропагаторы $D(t, 0) \in \mathbf{u}_{3}$ более общего вида могут быть построены в виде произведения пропагаторов, относящихся к различным представлениям $\mathbf{u}_{2}$ (см. п. 10.4).

10.3. Пред с та в лени я дин амики $\mathbf{u}_{2}$. Прилагаем алгебру Ли $\mathbf{u}_{3}{ }^{0}$ для составления списка всех представлений $\mathbf{u}_{2}{ }^{0}$ над $\mathbf{u}_{3}{ }^{0}$. Ищем сначала все такие базисы $Z, X, Y$ представлений $\mathbf{G}^{0}$ алгебры $\mathbf{u}_{2}{ }^{0}$, у которых $Z \in \mathbf{H}_{A}{ }^{0}$ (система представлений $\mathbf{G}$, примыкающих к $A$-базису).

Базисные операторы выбираем с одинаковой нормой

$$
(Z, Z)=(X, X)=(Y, Y)=\eta^{2}
$$

и с алгеброй Ли $\mathbf{u}_{2}{ }^{0}$

$$
-i[Z, X]=Y, \quad-i[Z, Y]=-X, \quad-i[X, Y]=Z .
$$

Алгебра Ли $\mathbf{u}_{3}{ }^{0}$ определяется следующей системой коммутационных соотношений:

$$
\begin{aligned}
& -i\left[H_{0}, X_{m n}\right]=\stackrel{0}{\omega_{m n}^{\prime} Y_{m n}^{\prime},} \\
& -i\left[H_{0}, Y_{m n}\right]=-\stackrel{0}{0} X_{m n}, \\
& -i\left[X_{m n}, Y_{m n}\right]=Z_{m n}, \\
& -i\left[X_{m k}, X_{k n}\right]=(1 / 2) Y_{m n}, \\
& -i\left[Y_{m k}, Y_{k n}\right]=-(1 / 2) Y_{m n}, \\
& -i\left[X_{m k}, Y_{k n}\right]=-(1 / 2) X_{m n}, \\
& -i\left[Y_{m k}, X_{k n}\right]=-(1 / 2) X_{m n} .
\end{aligned}
$$

Отметим попутно, что формулы $(10.39)-(10.45)$ остаются верными и 
в более общем случае алгебры Ли $\mathbf{u}_{d}{ }^{0}, d>3$. Для полноты характеристики $\mathbf{u}_{d}{ }^{0}$ следует добавить правило: базисные операторы несвязанных переходов коммутируют друг с другом.

Применение соотношений $(10.39)-(10.45)$ позволяет установить существование только двух типов вышеупомянутых представлений $\mathbf{G}^{0}$ (со свойством $\left.Z \in \mathbf{H}_{A}{ }^{0}\right)$ - типа $\mathbf{G}_{L}{ }^{0}(m n)$ и типа $\mathbf{G}^{0}(m k, k n)$. В случае $\mathbf{G}^{0}=\mathbf{G}_{L}{ }^{0}(m n)$ имеем $\eta^{2}=1 / 2, Z=Z_{m n}, X=X_{m n}, Y=Y_{m n}$. Так как $Q_{m n}$ коммутирует со всеми элементами $; \mathbf{G}_{L}{ }^{0}(m n)$, то включение его в список базисных операторов расширяет $\mathbf{G}_{L}{ }^{0}(\mathrm{mn})$ до 4-мерного приводимого представления $\mathbf{G}^{0}(\mathrm{mn})$.

Супероператорное представление соответствующей подгруппы $\mathbf{G}(\mathrm{mn})$ распадается на три неприводимых подпространства

$$
\mathbf{u}_{3}^{0}=\mathbf{S}^{0}(m n)+\mathbf{G}_{L}^{0}(m n)+\mathbf{H}(m k, k n),
$$

из которых 1-мерное $\mathbf{S}^{0}(m n)$ натянуто на $Q_{m n}$, 3-мерное $\mathbf{G}_{L}{ }^{0}(m n)-$ на $Z_{m n}, X_{m n}, Y_{m n}$, а 4-мерное $\mathbf{H}(m k, k n)$ опирается на $X_{m k}, Y_{m k}, X_{k n}$.и $Y_{k n}$.

В случае же $\mathbf{G}^{0}=\mathbf{G}^{0}(m k, k n)$ имеем $\eta^{2}=2$. Все базисы $Z, X, Y$ могут быть выведены исходя из базиса

$$
\begin{aligned}
& Z=2 Z_{m n}, \\
& X=X_{1}(m n)=\sqrt{2}\left(X_{m k}+X_{k n}\right), \\
& Y=Y_{1}(m n)=\sqrt{2}\left(Y_{m k}+Y_{k n}\right) .
\end{aligned}
$$

С этой целью следует воспользоваться преобразованием (10.9):

$$
\begin{aligned}
& X=\mathfrak{U}_{A} X_{1}(m n), \\
& Y=\mathfrak{U}_{A} Y_{1}(m n) .
\end{aligned}
$$

Частным случаем операторов (10.50) и (10.51) является пара

$$
\begin{aligned}
& X=X_{2}(m n)=\sqrt{2}\left(X_{m k}-X_{k n}\right), \\
& Y=Y_{2}(m n)=\sqrt{2}\left(Y_{m k}-Y_{k n}\right) .
\end{aligned}
$$

Четыре оператора $(10.48),(10.49),(10.52),(10.53)$ образуют ортогональный базис подпространства $\mathbf{H}(m k, k n)$.

Супероператорное представление подгруппы типа $\mathbf{G}(m k, k n)$ распадается на два неприводимых подпространства: на 3-мерное $\mathbf{G}^{0}(m k, k n)$ и на 5 -мерное ортогональное дополнение к нему. Представление изоморфно к тензорному представлению группы $\mathbf{d}_{3}$ (т. е. группы $S O(3))$ - подпространство $\mathbf{G}^{0}(m k, k n)$ преобразуется как 3-мерные евклидовые векторы, а его ортогональное дополнение - как неприводимые (евклидовые) тензоры второго ранга.

Подведем итоги. K $A$-базису примыкает целая система представлений динамики $\mathbf{u}_{2}$. Эта система распадается на подсистемы по трем переходам $m \rightarrow n$. Каждому базису $(10.1)$, (10.2) подпространства $\mathbf{H}_{A}{ }^{0}$ соответствует одна такая подсистема. Каждая подсистема состоит из поддинамики $\mathbf{G}(m n)$ и однопараметрического множества поддинамик типа $\mathbf{G}(m k, k n)$. Во главе всей системы - подпространство $\mathbf{H}_{A}{ }^{0}$.

Преобразование $(10.20)$ переводит систему представлений $\mathbf{G}$, примыкающих к $A$-базису, к системе представлений $\mathbf{G}_{T}$, примыкающих к $E^{(3)}$-базису, возглавляемому подпространством $\mathbf{H}_{F 3}^{0}$. Придавая оператору $T$ все «значения», допускаемые формулой $(10.10)$, получаем все 
множество представлений группы $\boldsymbol{u}_{2}$. Чтобы иметь все различные супероператоры, достаточно взять параметры Мойнихена в пределах

$$
-\pi / 2 \leqslant \varphi_{m n}, \sigma_{m n} \leqslant \pi / 2 .
$$

10.4. О бобщенны й дв ойн й резонанс. Рассмотрим движения, описываемые пропагаторами типа

$$
D(t, 0)=D_{1}(t, 0) D_{2}(t, 0) D_{3}(t, 0),
$$

где

$$
\begin{aligned}
& D_{1}(t, 0)=\exp \left(-i t G_{1}\right) \in \mathbf{G}(23), \\
& D_{2}(t, 0)=\exp \left(-i t G_{2}\right) \in \mathbf{G}(23), \mathbf{G}_{T}(12), \\
& D_{3}(t, 0)=\exp \left(-i t F_{2}\right) \in \mathbf{G}_{T}(12)
\end{aligned}
$$

Пропагатор (10.55) состоит из мультипликативных компонент (10.56)$(10.58)$, относящихся к поддинамикам $\mathbf{G}(23)$ и $\mathbf{G}_{T}(12)$. При этом компонента (10.57) принадлежит к обеим поддинамикам. Индекс $T$, в данном случае, означает $T=T_{23}$.

Пропагатору (10.55) соответствует супероператор

$$
\Re(t, 0)=\Re_{1}(t, 0) \Re_{2}(t, 0) \Re_{3}(t, 0),
$$

где $\Re_{j}(t, 0) \quad(j=1,2,3)$ - супероператор оператора $D_{j}(t, 0)$. суммы

Соответствующий прапагатору (10.55) гамильтониан имеет вид

$$
H(t)=G_{1}+\Re_{1}(t, 0) G_{2}+\Re_{1}(t, 0) \Re_{2}(t, 0) F_{2} .
$$

Если же из (10.63) выделить независящую от времени часть $H_{0} \in \mathbf{H}_{A}{ }^{0}$, то гамильтониан примет следующий вид:

где

$$
H(t)=H_{0}+H_{1}(t)+H_{2}(t),
$$

$$
\begin{aligned}
& H_{1}(t)=\Re_{1}(t, 0) V_{1}, \\
& H_{2}(t)=\Re_{1}(t, 0) \Re_{2}(t, 0) V_{2}, \\
& V_{1}=h_{1} X_{23}\left(\sigma_{23}\right) \in \mathbf{G}^{0}(23), \\
& V_{2}=h_{2} X_{12}^{(1)}\left(\sigma_{12}\right) \in \mathbf{G}_{T}^{0}(12) .
\end{aligned}
$$

Выберем $(10.64)-(10.68)$ за исходное определение гамильтониана и введем еще эрмитовый оператор

$$
F_{1}=H_{0}-G_{1}+V_{1}=\omega_{L}^{(1)} Z_{12}^{(1)}+\omega_{S}^{(1)} Q_{12}^{(1)} \in \mathbf{H}_{F 1}^{0} .
$$

Тогда частотные параметры операторов $F_{1}, F_{2}$ вычисляются по формулам

$$
\begin{aligned}
& \omega_{\mathrm{S}}^{(1)}=\omega_{\mathrm{S}}^{(2)}=(1 / 2) \Delta v_{s}+(3 / 4) \sqrt{\left(\Delta v_{1}\right)^{2}+h_{1}^{2}}, \\
& \omega_{L}^{(1)}=\Delta v_{s}-(1 / 2) \sqrt{\left(\Delta v_{1}\right)^{2}+h_{1}^{2}},
\end{aligned}
$$


таблица 3

Угловые частоты вращений, совершаемые супероператорами $\mathfrak{R}_{j}(t, 0)$

\begin{tabular}{c|c|c|c}
\hline $\begin{array}{c}\text { Переход } \\
m \rightarrow n\end{array}$ & $\mathfrak{k}_{1}(t, 0)$ & $\mathfrak{k}_{2}(t, 0)$ & $\mathfrak{K}_{3}(t, 0)$ \\
\hline $1 \rightarrow 2$ & $v_{S}-(1 / 2) v_{1}$ & $v_{2}$ & ${ }^{(2)}=\omega_{L}^{(2)}$ \\
$2 \rightarrow 3$ & $v_{1}$ & $-(1 / 2) v_{2}$ & $\omega_{12}^{(2)}=\omega_{S}^{(2)}-(1 / 2) \omega_{L}^{(2)}$ \\
$1 \rightarrow 3$ & $v_{S}+(1 / 2) v_{1}$ & $(1 / 2) v_{2}$ & $\omega_{23}^{(2)}=\omega_{S}^{(2)}+(1 / 2) \omega_{L}^{(2)}$
\end{tabular}

таблица 4

Характеристики оператора плотности (10.81)

\begin{tabular}{c|c|c|c}
\hline $\begin{array}{c}\text { Bра- } \\
\text { щенне }\end{array}$ & $v_{m n}^{q}$ & $\beta_{m n}^{q}$ & \multicolumn{1}{c}{$\varrho_{m n}^{q}$} \\
\hline \multirow{3}{*}{$X_{23} \rightarrow Y_{23}$} & $v_{1}$ & $\sigma_{23}$ & $(1 / 2) \varrho_{L}(0) \cos 2 \varphi_{13}-\varrho_{S}(0)$ \\
& $v_{1}-(1 / 2) v_{2}+\omega_{13}^{(2)}$ & $\sigma_{13}$ & $(1 / 2) \varrho_{L}(0) \sin 2 \varphi_{13} \sin \varphi_{12}\left(\cos 2 \varphi_{23}+1\right)$ \\
$X_{12} \rightarrow Y_{12}$ & $v_{1}+(1 / 2) v_{2}-\omega_{13}^{(2)}$ & $2 \sigma_{23}-\sigma_{13}$ & $(1 / 2) \varrho_{L}(0) \sin 2 \varphi_{13} \sin \varphi_{12}\left(\cos 2 \varphi_{23}-1\right)$ \\
& $v_{S}-(1 / 2) v_{1}+v_{2}$ & $\sigma_{12}$ & {$\left[(1 / 2) \varrho_{L}(0) \cos 2 \varphi_{13}+\varrho_{S}(0)\right] \sin 2 \varphi_{12} \cos \varphi_{23}$} \\
& $v_{S}-(1 / 2)\left(v_{1}-v_{2}\right)+\omega_{13}^{(2)}$ & $\sigma_{13}$ & $-Q_{L}(0) \sin 2 \varphi_{13} \cos \varphi_{12} \sin \varphi_{23}$ \\
$X_{13} \rightarrow Y_{13}$ & $v_{S}+(1 / 2) v_{1}+v_{2}$ & $\sigma_{12}$ & {$\left[(1 / 2) \varrho_{L}(0) \cos 2 \varphi_{13}+\varrho_{S}(0)\right] \sin 2 \varphi_{12} \sin \varphi_{23}$} \\
& $v_{S}+(1 / 2)\left(v_{1}+v_{2}\right)+\omega_{13}^{(2)}$ & $\sigma_{13}$ & $\varrho_{L}(0) \sin 2 \varphi_{13} \cos \varphi_{12} \cos \varphi_{23}$
\end{tabular}

где

$$
\omega_{L}^{(2)}=\sqrt{\left(\Delta v_{2}\right)^{2}+h_{2}^{2}}
$$

а углы $\varphi_{m n}$ - по формулам

$$
\begin{aligned}
& \Delta v_{S}=\omega_{S}^{0}-v_{S}, \\
& \Delta v_{1}=\omega_{L}^{0}-v_{1}, \\
& \Delta v_{2}=\omega_{L}^{(1)}-v_{2},
\end{aligned}
$$

Супероператор $\Re_{1}(t, 0)$ совершает врашение $X_{m n} \rightarrow Y_{m n} A$-базиса, оставляя $\mathbf{H}_{A}{ }^{0}$ неизменным. Аналогично, $\Re_{2}(t, 0)$ вращает $E^{(1)}$-базис, а $\Re_{3}(t, 0)-E^{(2)}$-базис. При этом неизменными остаются $\mathbf{H}_{F 1}^{0}$ и $\mathbf{H}_{F 2}^{0}$ соответственно (см. табл. 3).

Компоненты возбуждения (10.65) и (10.66) выражаются в виде

$$
\begin{gathered}
H_{1}(t)=h_{1}\left[\cos \left(v_{1} t+\sigma_{23}\right) X_{23}+\sin \left(v_{1} t+\sigma_{23}\right) Y_{23}\right], \\
H_{2}(t)=h_{2} \cos \varphi_{23}\left[\cos \left(v_{12} t+\sigma_{12}\right) X_{12}+\sin \left(v_{12} t+\sigma_{12}\right) Y_{12}\right]+ \\
+h_{2} \sin \varphi_{23}\left[\cos \left(v_{13} t+\sigma_{12}\right) X_{13}+\sin \left(v_{13} t+\sigma_{12}\right) Y_{13}\right],
\end{gathered}
$$

где

$$
v_{12}=v_{\mathrm{S}}-(1 / 2) v_{1}+v_{2}, \quad v_{13}=v_{\mathrm{S}}+(1 / 2) v_{1}+v_{2} .
$$

Оператор плотности задается формулой 


$$
\begin{aligned}
\varrho(t)=\varrho_{A}(t) & +\sum_{m<n} \sum_{q} \sum_{\varrho m} \underset{q}{q}\left[\cos \left(v_{m n}^{q} t+\beta_{m n}^{q}\right) X_{m n}+\right. \\
& \left.+\sin \left(v_{m n}^{q} t+\beta_{m n}^{q}\right) Y_{m n}\right]
\end{aligned}
$$

и параметрами, приведенными в табл. 4.

Компонента $\varrho_{A}(t) \in \mathbf{H}_{A}$ в формуле (10.81) имеет вид

$$
\begin{aligned}
\varrho_{A}(t)=(1 / 3) E & -\left[(3 / 4) \varrho_{L}(0) \cos 2 \varphi_{13}+(1 / 2) \varrho_{s}(0)\right] Q_{23}+ \\
& +\left[(1 / 2) \varrho_{L}(0) \cos 2 \varphi_{13}-\varrho_{s}(0)\right] Z_{23}- \\
& -\varrho_{L}(0) \sin 2 \varphi_{13} \sin \varphi_{12} \sin 2 \varphi_{23} \times \\
& \times \cos \left[\left(\omega_{13}^{(2)}-(1 / 2) v_{2}\right) t+\left(\sigma_{13}-\sigma_{23}\right)\right] Z_{23} .
\end{aligned}
$$

\section{ЛИ Т Е РА Т У РА}

1. С и н и в е э В., Изв. АН ЭССР, Физ. Матем., 24, № 1, 35-48 (1975).

2. Murn a g han, F. D., The unitary and rotation groups, Washington, Spartan books, 1962.

3. W e i, J., N orm a n, E., J. Math. Phys., 4, № 4, 575-581 (1963).

Ннститут кибернетики

Академии наук Эстонской ССР
Поступила в редакцию $4 /$ XII 1978

\section{SINIVEE}

\section{RUHMADE TEOORIA RAKENDAMISEST MITMESPINNISUSTEEMIDE DUNAAMIKA UURIMISEKS. VI}

Artiklis on käsitletud kolme nivooga kvantsüsteemi dünaamikat.

\section{SINIVEE}

\section{GROUP APPROACH IN DYNAMICS OF MANY-SPIN SYSTEMS. VI}

The dynamics of abstract three-level systems governed by a broad class of time-dependent Hamiltonians is considered. The propagators are constructed as products of exponentials. The corresponding time-dependent orthogonal transformations, which describe the motion of the density operator, are given in terms of rotating bases of the 9-dimensional space of hermitian operators. A list of subdynamics described by propagators which belong to representations of $S U(2)$ in $S U(3)$ is given. 